\title{
Corrigendum: Recognition and processing of the origin of transfer DNA by conjugative relaxase TrwC
}

\author{
Alicia Guasch, María Lucas, Gabriel Moncalián, Matilde Cabezas, Rosa Pérez-Luque, F Xavier Gomis-Rüth, Fernando de la Cruz \& \\ Miquel Coll \\ Nat. Struct. Biol. 10, 1002-1010 (2003).
}

The original version of this manuscript contained two mistakes. First, the concentrations of oligonucleotides quoted in the first sentence under the subheading "Electrophoresis mobility shift assay (EMSA)" in the Methods section were incorrect. The correct sentence should read: "Binding reactions contained $1 \mathrm{nM} 5^{\prime}$-radiolabeled oligonucleotide, $1 \mu \mathrm{M}$ competitor oligonucleotide...." Second, the PDB accession code for the $\mathrm{Zn}^{2+}$-bound TrwC-N293-DNA complex structure was reported incorrectly. The correct PDB accession code is 1QX0. The authors apologize for any inconvenience this may have caused.

\section{Addendum: Structure of a specific alcohol-binding site defined by the odorant binding protein LUSH from Drosophila melanogaster}

\author{
Schoen W Kruse, Rui Zhao, Dean P Smith \& David N M Jones \\ Nat. Struct. Biol. 10, 694-700 (2003).
}

A review by Dwyer and Bradley (cited as ref. 18 in the original article) presented an alignment of the alcohol-sensitive regions of several ion channels and proposed a consensus sequence motif for alcohol-binding sites. Kruse et al. acknowledge that the alignment of Dwyer and Bradley (Fig. 2 of ref. 18) bears superficial similarity to Figure 4 of their paper. Nevertheless, the authors state that the differences between the two are significant. Specifically, Figure 4 of this paper was constructed on the basis of an alignment with the high-resolution structures of LUSH-alcohol complexes. On the basis of this alignment the authors proposed a different consensus motif for alcohol-binding sites. As stated in the manuscript, the authors emphasize that sequence alignments alone are unlikely to define alcohol-binding motifs in these proteins; instead, the conformational proximity of the appropriate amino acids is likely to be more relevant. 